\title{
Tratamiento restaurador atraumático: Eficacia clínica de ionómeros de vidrio en cavidades clase I
}

\section{Atraumatic restorative treatment: Clinical efficacy of glass ionomers in class I cavities}

Katia Luz Medina Calderón', Jorge Raúl Colchado Carhuavilca², Luis Antonio Vicuña Huaqui ${ }^{3}$, Ezzard Omar Álvarez Díaz

1 Docente Asociado Dpto. Académico Estomatología Preventiva y Social. Doctorando en Esto matología. Facultad de Odontología, Universidad Nacional Mayor de San Marcos. Av. Carlos Ger mán Amézaga 375, Ciudad Universitaria, Lima 15081, Perú.

2 Docente Auxiliar Laboratorio Bioquímica. Doctorando en Estomatología. Facultad de Odonto logía, Universidad Nacional Mayor de San Marcos. Av. Carlos Germán Amézaga 375, Ciudad Universitaria, Lima 15081, Perú.

3 Estudiante de pregrado. Facultad de Odontología, Universidad Nacional Mayor de San Marcos Av. Carlos Germán Amézaga 375, Ciudad Universitaria, Lima 15081, Perú

4 Docente Principal Dpto. Académico Ing. de Sistemas e Informática. Facultad de Ingeniería Industrial. Universidad Nacional Mayor de San Marcos. Av. Carlos Germán Amézaga 375, Ciudad Universitaria, Lima 15081, Perú

Correspondencia:

Katia Luz Medina Calderón: kmedinac@unmsm.edu.pe, +51 942762291. Universidad Nacional Mayor de San Marcos. Av. Carlos Germán Amézaga 375, Ciudad Universitaria, Lima 15081, Perú

DOI: https:// 


\section{Resumen}

La Técnica de Restauración Atraumática (TRA) es un procedimiento preventivo-restaurador mínimamente invasivo, como alternativa de tratamiento para poblaciones vulnerables con poco o nulo acceso a servicios de salud, que consiste en la remoción del tejido dentario cariado utilizando sólo instrumentos manuales y un material adhesivo de restauración: el ionómero de vidrio de alta viscosidad debido a sus propiedades físicas y químicas, como la adhesión a la estructura dental, la biocompatibilidad, la reacción de fraguado químico y el desarrollo y la liberación de fluoruro, que le confieren características preventivas. Objetivo: Revisar la evidencia sobre la eficacia clínica, mediante el desempeño clínico y la supervivencia de los ionómeros en el tratamiento restaurador atraumático en dentición decidua. Métodos: Se realizó la búsqueda de información: estudios in vitro, ensayos clínicos aleatorizados y revisiones sistemáticas con antigüedad máxima de 10 años, en las bases de datos Medline, Scielo y Scopus, con las estrategias de búsqueda ("Dental Atraumatic Restorative Treatment/therapeutic use"[Mesh] OR "Dental Atraumatic Restorative Treatment/trends"[MesH] OR ART OR PRAT) y (("Dental Atraumatic Restorative Treatment"[Mesh]) AND ("Glass Ionomer Cements"[Mesh]) OR "Ketac-Molar Quick" [Supplementary Concept]). Conclusiones: Existe evidencia científica suficiente para determinar el éxito de las restauraciones TRA en lesiones de una superficie en dentición decidua, incluso en comparación con los materiales restauradores de los tratamientos convencionales. El desempeño clínico y la supervivencia es alta cuando se utilizan ionómeros de alta viscosidad. Aún no hay consenso acerca de las ventajas del uso de ionómeros de menor costo, encapsulados, reforzados con metal y/o con propiedades mejoradas.

Palabras clave: Tratamiento restaurador atraumático; cementos de ionómero vítreo; odontología pediátrica

\section{Abstract}

The Atraumatic Restoration Technique (ART) is a minimally invasive preventive-restorative procedure, as a treatment alternative for vulnerable populations with little or no access to health services, which consists of the removal of decayed dental tissue using only manual instruments and a restorative adhesive material: the glass ionomer of high viscosity due to its physical and chemical properties, such as adhesion to tooth structure, biocompatibility, chemical setting reaction and the development and release of fluoride, which confer preventive characteristics. Objective: To review the evidence on clinical efficacy through clinical performance and survival of ionomers in atraumatic restorative treatment in deciduous dentition. Methods: We searched for information: in vitro studies, randomized clinical trials and systematic reviews with a maximum age of 10 years, in the databases Medline, Scielo and Scopus, with search strategies ("Dental Atraumatic Restorative Treatment/therapeutic use"[Mesh] OR "Dental Atraumatic Restorative Treatment/trends"[MesH] OR ART OR PRAT) and (("Dental Atraumatic Restorative Treatment"[Mesh]) AND ("Glass lonomer Cements"[Mesh]) OR "Ketac-Molar Quick" [Supplementary Concept]). Conclusions: There is sufficient scientific evidence to determine the success of ART restorations in lesions of a deciduous dentition sur- 
face, even compared to the restorative materials of conventional treatments. Clinical performance and survival is high when high viscosity ionomers are used. There is still no consensus on the advantages of using lower-cost, encapsulated, metal-reinforced, and/or improved-properties- and/or lower-cost day-on-ones.

Key words: Atraumatic restorative treatment, vitreous ionomer cements, pediatric dentistry.

\section{Introducción}

En el Perú la caries dental es una de las enfermedades más prevalentes, $87 \%$ en niños menores a 12 años ${ }^{1}$ y constituye una de las principales causas de consulta en los establecimientos de salud, siendo más acentuada esta realidad en la población que se encuentra en condiciones de pobreza y bajos recursos económicos que no acceden a los servicios de salud bucal y en los casos que acuden optan por tratamientos de remoción quirúrgica de las piezas dentarias, afectando las funciones masticatorias, fonéticas, estéticas, entre otras. ${ }^{2}$

Ante esta problemática de falta de acceso a tratamientos iniciales primarios de abordaje de la caries dental y limitación del daño se han adoptado enfoques mínimamente invasivos en población infantil y con desventaja social, mediante el tratamiento restaurador atraumático (TRA), el cual muestra evidencia consistente de adecuados resultados en comparación con la técnica tradicional; esta técnica emplea conocimientos sobre remoción parcial de caries, dentina desmineralizada, con el enfoque de la Odontología mínimamente invasiva. ${ }^{3-4}$

El material empleado para la restauración con esta técnica es el ionómero de vidrio, el cual resalta en altas tasas de supervivencia debido a sus propiedades de adhesión a tejido dentario, liberación de flúor, biocompatibilidad, propiedad térmica y mecánica, solubilidad, estética y manipulación. ${ }^{4}$ Diversos estudios de evaluación de supervivencia han permitido determinar el tiempo y el desempeño clínico que el ionómero permanece en la cavidad bucal; sin embargo, es necesario seguir evaluando otra propuesta de marca alternativa de menor costo que permitan emplearse en la salud pública, por ello, el objetivo de este estudio es revisar la evidencia disponible sobre la eficacia clínica y aceptabilidad en el tiempo de los ionómeros de vidrio en restauraciones clase I de piezas deciduas.

\section{Metodología}

Se realizó la búsqueda de información en las bases de datos Medline, Scielo y Scopus, con las palabras claves ("Dental Atraumatic Restorative Treatment/therapeutic use "[Mesh] OR "Dental Atraumatic Restorative Treatment/trends"[MesH] OR ART OR PRAT) y (("Dental Atraumatic Restorative Treatment"[Mesh]) AND ("Glass lonomer Cements"[ Mesh]) OR "Ketac-Molar Quick" [Supplementary Concept]). Se limitó a estudios de 10 años la búsqueda de las investigaciones, se incluyó estudios in vitro, ensayos clínicos aleatorizados y revisiones sistemáticas, sin restricción de idiomas y espacio geográfico. 


\section{Estado del Arte}

Técnica de Restauración Atraumática

La caries dental es una enfermedad considerada como una "disbiosis" manifestada por el elevado consumo de azúcares fermentables que provoca un desequilibrio en la proporción de los microorganismos y eventos metabólicos del biofilm oral, que generan una posterior disolución química localizada de la superficie dentaria conocido como proceso carioso; ${ }^{5}$ para disminuir el riesgo de aparición de nuevas lesiones, el control de la placa, la alimentación bajo en azúcares y la atención oportuna con el odontólogo son medidas fundamentales. ${ }^{6}$

Como parte de estas medidas, se implementó el uso del TRA en la atención en poblaciones de zonas marginadas y de difícil acceso: ${ }^{7}$ el enfoque TRA se basa en el tratamiento de dientes cariados mediante la eliminación de la dentina afectada con instrumentos de mano y sin administración de anestesia local, ${ }^{8}$ es un procedimiento alternativo de bajo costo al prescindir de tecnología convencional y se encuentra basado en el paradigma de la odontología mínimamente invasiva. ${ }^{9}$ Se presenta como un proceso agradable, brinda gran expectativa para su uso en niños y adultos temerosos y en pacientes con impedimento físico y mental, que viven en casas de reposo y abandonadas. ${ }^{10}$

\section{El ionómero de vidrio para el Tratamiento Restaurador Atraumático}

El ionómero de vidrio es un material innovador al presentar como ventaja la liberación de fluoruro, adhesión específica a esmalte y dentina y coeficiente de expansión térmica similar al diente (11) emergió como un material restaurador a principios de la década de 1970, con estética más agradable que las restauraciones metálicas; sin embargo, en áreas donde debían soportar estrés era una limitante, dado las pobres propiedades mecánicas como la baja resistencia a la fractura, tenacidad y resistencia al desgaste. ${ }^{12-13}$

Con el paso del tiempo, las propiedades han sido mejoradas y varios factores llevaron a la selección del ionómero de vidrio como un adecuado material para el TRA como la liberación de fluoruro hasta por cinco años, depósito de la absorción de iones de fluoruro tópico(14)(15) adhesión química al esmalte y a la dentina a través del intercambio iónico, biocompatibilidad con los tejidos orales, naturaleza soluble, alta resistencia a la abrasión buena humectación y rápida aplicación. ${ }^{9-10-16}$

En la actualidad, en el TRA se emplean con mayor frecuencia ionómeros de alta viscosidad como Fuji IX (GC) y Ketac Molar (3M ESPE) y en menor medida, los de baja viscosidad como Chemfil (Dentsply), Chemflex (Dentsply) y Fuji Plus (GC Int). ${ }^{2-14}$ El ionómero de vidrio Ketac ${ }^{\text {TM }}$ Molar Easymix fue desarrollado para un mezclado más rápido, una dosificación más precisa, una mejor humectación del polvo por el líquido y una menor producción de polvo durante mezclado; esto se logró mediante la granulación del polvo de Ketac Molar para que las propiedades mecánicas y liberación de iones de flúor no fueran afectadas de manera negativa, otorgándole de esta forma una alta proporción de polvo a líquido y mejora en la resistencia al desgaste, compresión, y adaptabilidad marginal..$^{14}$ 
Eficacia clínica según la supervivencia y desempeño clínico de las restauraciones con el Tratamiento Restaurador Atraumático

La literatura científica reporta como eficacia clínica la medida en la que un individuo, en una población definida, se beneficie de la resolución de un problema de salud, determinado bajo condiciones ideales de actuación, en el TRA es definido como beneficios para los preescolares después del procedimiento restaurativo, los cuales pueden calificarse como éxito o fracaso según la supervivencia y desempeño clínico. ${ }^{17-18}$

La supervivencia hace referencia al estado de la restauración realizada con el Tratamiento Restaurador Atraumático (TRA), en las cavidades de molares deciduas o permanentes posterior a cierto tiempo, para dicho fin se utilizan los códigos y criterios de calificación de Frencken et al (1996) $)^{19}$ (Gráfico 1)

\begin{tabular}{|c|c|}
\hline \multicolumn{2}{|r|}{ Supervivencia de restauraciones TRA según Frencken et al (1996) (19) } \\
\hline Código & Criterio \\
\hline 0 & Presente, bueno \\
\hline 1 & $\begin{array}{l}\text { Presente, defecto marginal y/o desgaste en la superficie leve, el cual es } \\
\text { menor de } 0.5 \mathrm{~mm} \text { de profundidad: no es necesario cambiar o reparar la } \\
\text { restauración }\end{array}$ \\
\hline 2 & $\begin{array}{l}\text { Presente, defecto marginal y/o desgaste en la superficie, el cual es más } \\
\text { profundo que } 0.5 \mathrm{~mm} \text { pero menor de } 1.0 \mathrm{~mm} \text { : es necesario reparar la restau- } \\
\text { ración }\end{array}$ \\
\hline 3 & $\begin{array}{l}\text { Presente, defecto marginal y/o desgaste en la superficie grosero, de más } \\
\text { de } 1.0 \mathrm{~mm} \text { de profundidad: es necesario reparar la restauración }\end{array}$ \\
\hline 4 & $\begin{array}{l}\text { Ausente, la restauración ha desaparecido completamente (o la mayor par- } \\
\text { te): es necesario un nuevo tratamiento. }\end{array}$ \\
\hline 5 & Ausente, se ha realizado otro tratamiento restaurador \\
\hline 6 & Ausente, el diente ha sido extraído \\
\hline 7 & $\begin{array}{l}\text { Presente, extensas áreas de la restauración se han desgastado gradual- } \\
\text { mente pero menos de } 0.5 \mathrm{~mm} \text { en el punto de mayor profundidad: no es } \\
\text { necesario la reparación de la restauración }\end{array}$ \\
\hline 8 & $\begin{array}{l}\text { Presente, extensas áreas de la restauración se han desgastado más de } \\
0.5 \mathrm{~mm} \text { de profundidad: es necesario la reparación de la restauración }\end{array}$ \\
\hline 9 & Imposible de diagnosticar \\
\hline
\end{tabular}


El desempeño clínico es la evaluación de características clínicas de la restauración como retención, color, integridad de los márgenes, forma anatómica, textura superficial, decoloración de los márgenes y caries recurrente, posterior a cierto tiempo de haber sido realizada el TRA en cavidades Clase I de molares deciduas superiores o inferiores posterior, empleando el criterio de USPHS modificado y calificándolos en Alpha A (Éxito ideal), Bravo B (Éxito satisfactorio) y Charlie $C$ y Delta $D$ (Falla insatisfactorio)..$^{7-18}$

\section{Factores que afectan el éxito del Tratamiento Restaurador Atraumático}

El éxito del Tratamiento Restaurador Atraumático dependerá del adecuado diagnóstico y la selección de la pieza dentaria a restaurar, el fracaso es atribuido a muchos factores entre ellos la limitada experiencia y entrenamiento del profesional 10, la remoción insuficiente de caries, acondicionamiento inadecuado de la cavidad e inapropiado mezclado del material (polvo/líquido); sin embargo, con mayor frecuencia estas condiciones no se registran en los estudios de seguimiento, solo se reportan tipos de fallas como la pérdida parcial o total de la restauración y diferentes tipos de desgaste o fracturas que requerirán reparación o reemplazo. ${ }^{3-20-21-22}$

Otros factores en el fracaso de las restauraciones son los relacionados al paciente, como la contaminación por saliva o sangre, tipo de aislamiento, nivel de cooperación del paciente niño, número de restauraciones por niño y falta de cumplimiento de las indicaciones post tratamiento como no ingerir líquidos o alimentos una hora después ${ }^{3-2}$ no obstante, Hilgert y col (2014) ${ }^{23}$ reportan la no existencia de diferencias significativas en los resultados de las restauraciones a pesar de haber utilizado aislamiento relativo con rollos de algodón, Roshan y Sakeenabi (2011)11, Abbass MMS y col (2019) ${ }^{21}$ consideran las limitaciones de la estructura morfológica 0 anatómica de los dientes deciduos, fallas en el control de la saliva en los niños y la susceptibilidad de las restauraciones superficiales o poco profundas, Hesse D y col (2016)22, Abbass MMS y col (2019) ${ }^{21}$ indican como relevante el factor personal o "factor operador" como la formación académica, el nivel de experiencia, el entrenamiento en el protocolo, la posición del operador y del paciente, la iluminación, el confort del operador y la accesibilidad para la eliminación de la caries, Frencken y col (2013) ${ }^{24}$ atribuyen las fallas a las características físicas del ionómero y el manejo del material por el operador.

Según Luengas-Quintero y col $(2013)^{25}$ y van Gemert-Schriks y col $(2007)^{26}$ los factores ambientales de la zona donde se realiza el procedimiento restaurativo como la temperatura ambiental, humedad atmosférica, la altitud y la localización geográfica influyen en la velocidad de la preparación y en la calidad del ionómero, en consecuencia en la supervivencia de las restauraciones. Se han reportado otros factores como: la posición del diente en las arcadas (derecho o izquierdo) y la superficie a restaurar (oclusal, vestibular, palatino) que no tienen influencia en la sobrevida de las restauraciones TRA en dientes deciduos. ${ }^{25}$

También, se señala que la experiencia de caries (ceod, CPOD) es un factor directamente relacionado al riesgo de desarrollar nuevas lesiones. Sin embargo, Pacheco y col. ${ }^{27}$ no encontraron diferencias significativas entre los grupos de alto, mediano o bajo nivel de experiencia de caries, debido a que previo a las restauraciones los niños y sus padres reciben actividades educativas sobre higiene oral y otros temas de prevención ${ }^{13-20-22}$ 


\section{Discusión}

En los reportes de la literatura científica existen resultados diversos respecto a la supervivencia de los tratamientos basados en el TRA, por ejemplo van Gemert-Schriks MC y col $(2007)^{26}$, Lo $E$ y col $(2001)^{28}$ reportan, en un tiempo breve, resultados de éxito de 80 a $95 \%$ en una sola superficie (clases I y V), de 55 a $75 \%$ en la Clase II y 32 a $55 \%$ en las clases III y IV, Saber y col (2019) 4 en un meta-análisis desarrollado por Van't Hof y col en 2006 reportaron supervivencia del $95 \%$ en dentición decidua y $97 \%$ para dentición permanente a los 12 meses de evaluación en lesiones de una superficie utilizando un ionómero de alta viscosidad, otro autores concluyen que la duración es significativamente más baja en lesiones proximales que en lesiones oclusales..$^{14}$

De Amorin y col (2012 $)^{29}$ en un meta-análisis no encontraron diferencias significativas en la supervivencia en reportes entre los años 2005 y 2010 y una mayor retención en boca se debía al empleo de ionómero de vidrio de alta viscosidad, por el cual se hace ventajoso utilizar este material en restauraciones y sellantes, Pacheco y col $(2017)^{27}$ hallaron tasa de sobrevida de $42,74 \%$, en un año de seguimiento, en el tratamiento de lesiones de caries ocluso proximales en dientes primarios, da Franca y col $(2013)^{9}$ reportaron éxito de $94,6 \%$ en un mes y $50,6 \%$ a un año en restauraciones de una superficie y $70,1 \%$ de sobrevida en el primer mes de lesiones restauradas de clase 1 .

Existen en el mercado nuevos productos, algunos de los cuales son producidos en Brasil, como el Vitromolar (Nova DFL) que al ser comparado con el Ketac Molar (3M ESPE), no presenta diferencias significativas en la calidad de sus propiedades y presenta una supervivencia de $34,5 \%$ en restauraciones ocluso-proximales. Pacheco y col $(2017)^{27}$ en la evaluación de otras marcas de ionómeros de bajo costo, reportaron heterogeneidad de $31 \%$ y $100 \%$ en la supervivencia de restauraciones ocluso proximales. ${ }^{14}$

Olegario et al (2016) 10 evaluaron el desempeño a 12 meses de ionómeros de vidrio de las marcas GC Gold Label 9, VitroMolar y Maxxion R, reportándose sobrevida de 77,5\%, 61,1\% y $57.5 \%$ respectivamente, donde el primero mostró mejores resultados probablemente por el nivel de rugosidad del material. De Lima Navarro et al (2007)7 investigaron en infantes de 18 a 36 meses de vida la sobrevida del TRA con los materiales Ketac Molar Easy Mix ${ }^{\circledR}$ (3M ESPE) y Vitro Molar ${ }^{\circledast}(\mathrm{DFL})$, obteniéndose éxito en el $94,8 \%, 87,9 \%$ y $82,3 \%$ en 1,2 y 4 años de seguimiento respectivamente y añade resultado de un meta-análisis de 18 países donde hallaron tasa de fallas en piezas molares deciduas para superficies únicas de 3,5\% y de $19 \%$ en superficies múltiples.

De Medeiros et al $(2017)^{3}$ observaron después de 12 meses una tasa de éxito de 89,3\% sin diferencias estadísticamente significativas entre el ionómero Ketac Molar Easy Mix 3M ESPE y la resina Filtek Z250-3M ESPE, Lo y Holmgren (2001)28 reportaron después de 30 meses de seguimiento, supervivencia de $79 \%$ en clase l y $70 \%$ en clase $V$, Honkala et al $(2003)^{30}$ después de 24 meses de seguimiento, mostraron éxito en Clase I de $89.6 \%$ sin diferencia estadística con restauraciones de amalgama, Frencken et al $(1996)^{19}$ en un meta-análisis evidenciaron que 
las superficies tratadas con amalgama presentaron mayor durabilidad que las restauradas con TRA solo durante los primeros años, a los tres años no se encontraron diferencias estadísticamente significativas, Holmgren y col (2000) ${ }^{8}$ en una evaluación del Ketac Molar encontraron supervivencia en restauraciones Clase I de $91 \%$ y $79 \%$, Clase V de $79 \%$ y $70 \%$, y Clase II $75 \%$ y $51 \%$ para dos evaluaciones de 12 y 30 meses, respectivamente para cada caso, Luengas-Quintero y col. $(2013)^{25}$ hallaron que luego de 12 y 24 meses los rangos de supervivencia de restauraciones de una superficie fueron $82,1 \%$ y $74 \%$ respectivamente. Roshan et al (2011) compararon la sobrevida de restauraciones TRA en dentición decidua colocadas en la escuela y en un hospital, encontrando el $82,2 \%$ de éxito después de seis meses y $77,7 \%$ después de 12 meses en las restauraciones colocadas en escuela y del $87,7 \%$ y $81,5 \%$ a los 6 y 12 meses en las restauraciones colocadas en el hospital.

Faustino-Silva \& Figueiredo y col. (2019) $)^{31}$ en ECA a doble ciego y boca dividida analizaron la efectividad del TRA y compararon Ketac Molar Easymix ${ }^{\circledR}$ (3M ESPE) y Vitro Molar ${ }^{\circledast}$ (DFL) a 1, 2 y 4 años en molares deciduos. A un 1 año se controló 100 piezas con un éxito de $94 \%$, a los 2 años 80 piezas con un éxito de $87,5 \%$ y a los 4 años éxito final de $82,9 \%$; sin diferencias entre las dos marcas de ionómeros $(p>0,05)$ según el criterio USPHS.

Freitas et al. (2018)32 realizaron un ECA de boca dividida para el rendimiento clínico de ionómeros de vidrio Riva Self-Cure (SDI Limited) Bayswater (VIC Australia) que son administrados en kits de mezcla manual y de forma encapsulada en cavidades clase I, donde hubo una tasa de éxito de $86,2 \%$ en el rendimiento clínico del ionómero encapsulado. Menezes-Silva et al. $(2019)^{17}$ analizaron la efectividad de las restauraciones con resina compuesta frente al TRA en lesiones clase II, empleando Equia Fil-GC Corporation y Filtek Z350 XT Universal Restorative, posterior a 12 meses el éxito del TRA fue de 95,8\% y 98,7\% para la resina, según criterios TRA y USPHS modificado, no existiendo diferencia significativa estadísticamente.

Olegário et al. (2016) $)^{10}$ evaluaron la eficacia en restauraciones de una y más superficies realizados con Ketac Molar Easy Mix (3M/ESPE) con TRA y tratamiento convencional con Filtek Bulk Fill (3M/ESPE), después de 24 meses, encontrando similitud en la efectividad de ambos, pero un mejor costo efectividad del TRA; Mickenautsch \& Yengopal y col (2015) ${ }^{33}$ realizaron una revisión sistemática, con la finalidad de responder si existía superioridad de las restauraciones con cemento de ionómero de vidrio de alta viscosidad (CIVAV) frente a la técnica convencional (TC) en superficies únicas o múltiples de piezas permanentes posteriores, concluyendo que no existe evidencia de la superioridad del CIVAV sobre el TC.

Pacheco et al (2017)27 realizaron un ECA para evaluar la supervivencia a 12 meses del Ketac Molar EasyMix (3M ESPE) y Vitromolar (DFL) en restauraciones ocluso proximales realizadas con TRA, la supervivencia del Ketac Molar fue 50,85\% y 34,48\% del Vitro Molar ( $p>0,058$ ), concluyendo que no existe diferencia significativa en el rendimiento clínico entre ambos ionómeros. Mobarak et al. (2019)34 desarrollaron un ECA a triple ciego para comparar la eficacia clínica entre un ionómero reforzado con zinc (ChemFil Rock) y ionómero de vidrio de alta viscosidad (Fuji IX GP) a 2 años, la sobrevida del Fuji IX GP fue 95,4\% y 85.3\% del ChemFil Rock, ( $p=0,02)$. Silva de Moura et al. (2020) 35 compararon a 12 meses el rendimiento entre un cemento ionó- 
mero de vidrio de bajo costo (Vitro Molar ${ }^{\circledR}$ ) y el cemento ionómero de vidrio de alta viscosidad (Ketac Molar ${ }^{\circledR}$ ), en niños entre 2 y 6 años que presentaron lesiones de clase I, II, III o IV; los resultados mediante los criterios TRA fueron supervivencia del 82,2\% en Ketac Molar ${ }^{\oplus}$ y 73,4\% en Vitro $\operatorname{Molar}^{\oplus}(p=0,011)$ y mayor prevalencia en superficies pequeñas $(p=0,001)$, medianas $(p=0,005)$ y en el segundo molar $(p=0,0015)$, evidenciando que Vitro Molar ${ }^{\oplus}$ presenta menor éxito en comparación al Ketac Molar ${ }^{\circledR}$.

D'Costa et al. (2020)36 buscaron comparar la longevidad a 18 meses del CIVAV GC Gold Label 9 y el ionómero de vidrio reforzado con metal GC Mirale Mix ${ }^{\oplus}$ empleados en TRA, el éxito del GC Mirale Mix ${ }^{\circledR}$ fue de $87,2 \%$ y del GC Gold Label 9 fue $61,8 \%$ ( $\left.p=0,015\right)$ concluyendo que el ionómero de vidrio reforzado con metal presenta mayor éxito respecto al ionómero de vidrio convencional.

\section{Conclusiones}

Se ha demostrado altas tasas de éxito, mediante el desempeño clínico y la supervivencia de las restauraciones TRA para las lesiones de caries de la dentición primaria. La literatura muestra una gran heterogeneidad en las tasas de supervivencia de las restauraciones TRA ocluso-proximal en dientes primarios después de un año, pero se han encontrado altas tasas de éxito en lesiones de una superficie de piezas deciduas y permanentes con TRA que oscilan entre el $90-100 \%$ en períodos de 12 meses a más.

Los cementos de ionómero de vidrio de alta viscosidad se han desarrollado especialmente para el TRA, debido a que tienen propiedades mecánicas mejoradas, lo que resulta en una mayor longevidad y desempeño clínico de las restauraciones, tanto en dientes permanentes como en deciduos. Las tasas de supervivencia de los materiales restauradores utilizados con TRA dependerá de diversos factores, entre ellos: la experiencia y entrenamiento de los profesionales para lograr elevados índices de éxito, o la marca del ionómero seleccionado, por sus propiedades establecidas.

Una serie de marcas en el mercado, algunos producidos en Brasil y vendidos en América Latina, también son indicados para el TRA con un menor costo. Sin embargo, el desempeño clínico y la supervivencia es alta cuando se utilizan ionómeros de alta viscosidad. Aún no hay consenso acerca de las ventajas del uso de ionómeros de menor costo, encapsulados, reforzados con metal y/o con propiedades mejoradas. 


\section{Referencias Bibliográficas}

1. Ministerio de Salud. Prevalencia Nacional de caries dental, fluorosis del esmalte y urgencia de tratamiento en escolares de 6 a 8, 10, 12 y 15 años. Perú. 2001 - 2002 - 2005.

2. Jurasic MM, Marianne Jurasic M, Gibson G, Wehler CJ, Orner MB, Jones JA. Caries prevalence and associations with medications and medical comorbidities [Internet]. Vol. 79, Journal of Public Health Dentistry. 2019. p. 34-43. Available from: http://dx.doi.org/10.1111/ jphd.12292

3. de Medeiros Serpa EB, Clementino MA, Granville-Garcia AF, Rosenblatt A. The effect of atraumatic restorative treatment on adhesive restorations for dental caries in deciduous molars. J Indian Soc Pedod Prev Dent. 2017 Apr;35(2):167-73.

4. Saber A, El-Housseiny A, Alamoudi N. Atraumatic Restorative Treatment and Interim Therapeutic Restoration: A Review of the Literature [Internet]. Vol. 7, Dentistry Journal. 2019. p. 28. Available from: http://dx.doi.org/10.3390/dj7010028

5. Frencken JE, van 't Hof MA, van Amerongen $W E$, Holmgren CJ. Effectiveness of Single-surface ART Restorations in the Permanent Dentition: A Meta-analysis [Internet]. Vol. 83, Journal of Dental Research. 2004. p. 120-3. Available from: http://dx.doi. org/10.1177/154405910408300207

6. Estupiñán-Day S, Tellez M, Kaur S, Milner T, Solari A. Managing dental caries with atraumatic restorative treatment in children: successful experience in three Latin American countries. Rev Panam Salud Publica. 2013 Apr;33(4):237-43.

7. Navarro MF de L, Leal SC, Molina GF, Villena RS. Tratamento Restaurador Atraumático: atualidades e perspectivas. Rev Assoc Paul Cir Dent. septiembre de 2015;69(3):289-301.

8. Holmgren CJ, Lo ECM, Hu D, Wan H. ART restorations and sealants placed in Chinese school children - results after three years [Internet]. Vol. 28, Community Dentistry and Oral Epidemiology. 2000. p. 314-20. Available from: http://dx.doi.org/10.1034/j.16000528.2000.280410.x

9. da Franca C, Colares V, Van Amerongen E. Two-year evaluation of the atraumatic restorative treatment approach in primary molars class I and II restorations. Int J Paediatr Dent. $2011 \mathrm{Jul} ; 21(4): 249-53$

10. Olegário IC, de Brito Pacheco AL, de Araújo MP, de Miranda Ladewig N, Bonifácio CC, Imparato JCP, et al. Low-cost GICs reduce survival rate in occlusal ART restorations in primary molars after one year: A RCT [Internet]. Vol. 57, Journal of Dentistry. 2017. p. 45-50. Available from: http://dx.doi.org/10.1016/j.jdent.2016.12.006

11. Noor-Mohammed R, Basha S. Survival of Occlusal ART Restorations in Primary Molars 
Placed in School Environment and Hospital Dental Setup-One Year Follow-up Study [Internet]. Medicina Oral Patología Oral y Cirugia Bucal. 2011. p. e973-7. Available from: http://dx.doi. org/10.4317/medoral.17327

12. Delgado-Angulo EK, Ortiz EB, Sánchez-Borjas PC. Análisis de supervivencia de sellantes y restauraciones ART realizados por estudiantes de pregrado [Internet]. Vol. 15, Revista Estomatológica Herediana. 2014. p. 119. Available from: http://dx.doi.org/10.20453/reh. v15i2.1941

13. Hosoya Y, García-Godoy F. Bonding mechanism of Ketac-Molar Aplicap and Fuji IX GP to enamel and dentin. Am J Dent. 1998 0ct;11(5):235-9.

14. Menne-Happ U, Ilie N. Effect of heat application on the mechanical behaviour of glass ionomer cements [Internet]. Vol. 18, Clinical Oral Investigations. 2014. p. 643-50. Available from: http://dx.doi.org/10.1007/s00784-013-1005-4

15. Frencken JE, Leal SC, Navarro MF. Twenty-five-year atraumatic restorative treatment (ART) approach: a comprehensive overview. Clin Oral Investig. 2012 0ct;16(5):1337-46.

16. Araujo MP, Innes NP, Bonifácio CC, Hesse D, Olegário IC, Mendes FM, et al. Atraumatic restorative treatment compared to the Hall Technique for occluso-proximal carious lesions in primary molars; 36-month follow-up of a randomised control trial in a school setting. BMC Oral Health. 2020 Nov 11;20(1):1-18.

17. Menezes-Silva, R., Velasco, S.R.M., Bastos, R.S. et al. Randomized clinical trial of class II restoration in permanent teeth comparing ART with composite resin after 12 months. Clin Oral Invest 23, 3623-3635 (2019). https://doi.org/10.1007/s00784-018-2787-1

18. Zanata RL, Fagundes TC, Freitas MCC de A, Lauris JRP, Navarro MF de L. Ten-year survival of ART restorations in permanent posterior teeth. Clin Oral Investig. 2011 Apr;15(2):265-71.

19. Frencken JE, Pilot T, Songpaisan Y, Phantumvanit P. Atraumatic restorative treatment (ART): rationale, technique, and development. J Public Health Dent. 1996;56(3 Spec):135-40; discussion 161-3.

20. Dye BA, Hsu K-LC, Afful J. Prevalence and Measurement of Dental Caries in Young Children. Pediatr Dent. 2015 May;37(3):200-16.

21. Abbass MMS, Mahmoud SA, El Moshy S, Rady D, AbuBakr N, Radwan IA, et al. The prevalence of dental caries among Egyptian children and adolescences and its association with age, socioeconomic status, dietary habits and other risk factors. A cross-sectional study. F1000Res. 2019 Jan 3;8:8.

22. Hesse D, de Araujo MP, Olegário IC, Innes N, Raggio DP, Bonifácio CC. Atraumatic Restorative Treatment compared to the Hall Technique for occluso-proximal cavities in primary molars: study protocol for a randomized controlled trial. Trials. 2016 Mar 31;17:169. 
23. Hilgert LA, de Amorim RG, Leal SC, Mulder J, Creugers NHJ, Frencken JE. Is high-viscosity glass-ionomer-cement a successor to amalgam for treating primary molars? Dent Mater. 2014 0ct;30(10):1172-8.

24. Frencken JEFM, Flohil KA, de Baat C. [The history and scientific development of atraumatic restorative treatment]. Ned Tijdschr Tandheelkd. 2013 Dec;120(12):677-81.

25. Luengas-Quintero E, Frencken JE, Muñúzuri-Hernández JA, Mulder J. The atraumatic restorative treatment (ART) strategy in Mexico: two-years follow up of ART sealants and restorations. BMC Oral Health. 2013 Sep 8;13:42.

26. van Gemert-Schriks MCM, van Amerongen WE, ten Cate JM, Aartman IHA. Three-year survival of single- and two-surface ART restorations in a high-caries child population. Clin Oral Investig. 2007 Dec;11(4):337-43.

27. Anna Luisa de Brito P, Isabel Cristina 0, Clarissa Calil B, Ana Flávia Bissoto C, José Carlos Pettorossi I, Daniela Prócida R. One year Survival Rate of Ketac Molar versus Vitro Molar for Occlusoproximal ART Restorations: a RCT. Braz Oral Res. 2017 Nov 6;31:e88.

28. Lo EC, Holmgren CJ. Provision of Atraumatic Restorative Treatment(ART) restorations to Chinese pre-school children-a 30-month evaluation. Int J Paediatr Dent. 2001 Jan;11(1):310.

29. de Amorim RG, Leal SC, Frencken JE. Survival of atraumatic restorative treatment (ART) sealants and restorations: a meta-analysis. Clin Oral Investig. 2012 Apr;16(2):429-41.

30. Honkala E, Behbehani J, Ibricevic H, Kerosuo E, Al-Jame G. The atraumatic restorative treatment (ART) approach to restoring primary teeth in a standard dental clinic. Int J Paediatr Dent. 2003 May;13(3):172-9.

31. Faustino-Silva DD, Figueiredo MC. Atraumatic restorative treatment-ART in early childhood caries in babies: 4 years of randomized clinical trial. Clin Oral Investig. 2019 Oct;23(10):3721-9.

32. Freitas MCC de A, Fagundes TC, Modena KC da S, Cardia GS, Navarro MF de L. Randomized clinical trial of encapsulated and hand-mixed glass-ionomer ART restorations: one-year follow-up. J Appl Oral Sci [Internet]. 2018 Jan 18 [cited 2021 Aug 14];26. Available from: http:// www.scielo.br/j/jaos/a/wkvmgDxKRMxL4kbZ8tYQ43y/?lang=en\&format=pdf

33. Mickenautsch S, Yengopal V. Failure Rate of Direct High-Viscosity Glass-Ionomer Versus Hybrid Resin Composite Restorations in Posterior Permanent Teeth - a Systematic Review. Open Dent J. 2015 Dec 22;9:438-48.

34. Mobarak E, El-Deeb H, Daifalla LE, Ghaly M, Mustafa M, Sabry D, et al. Survival of multiple-surface ART restorations using a zinc-reinforced glass-ionomer restorative after 2 years: A randomized triple-blind clinical trial. Dent Mater. 2019 Sep;35(9):e185-92. 
35. Moura MS de, Sousa GP de, Brito MHSF, Silva MCC, Lima M de DM de, Moura L de FA de D, et al. Does low-cost GIC have the same survival rate as high-viscosity GIC in atraumatic restorative treatments? A RCT. Braz Oral Res. 2020 Jan 24;33:e125.

36. D'Costa VG, Singhal DK, Acharya S. Efficacy of GC Gold Label 9 and GC Miracle Mix ${ }^{\circledR}$ Restorations using Atraumatic Restorative Treatment (ART) in Rural Settings: A Randomized Controlled Trial. J Clin Pediatr Dent. 2020 Jan 1;44(3):148-53. 